\title{
Anyonic Quantum Walks
}

\author{
Gavin K. Brennen ${ }^{1}$, Demosthenes Ellinas ${ }^{2}$, Viv Kendon ${ }^{3}$, \\ Jiannis K. Pachos ${ }^{3}$, Ioannis Tsohantjis ${ }^{2}$, and Zhenghan Wang ${ }^{4}$ \\ ${ }^{1}$ Centre for Quantum Information Science and Security, Macquarie University, 2109, NSW Australia \\ ${ }^{2}$ Department of Sciences, Division of Mathematics, \\ Technical University of Crete, GR - 73 100, Chania, Crete, Greece \\ ${ }^{3}$ School of Physics and Astronomy, University of Leeds, Leeds LS2 9JT, UK and \\ ${ }^{4}$ Microsoft Research, Station Q, University of California, Santa Barbara, CA 93106, USA
}

(Dated: August 28, 2018)

\begin{abstract}
The one dimensional quantum walk of anyonic systems is presented. The anyonic walker performs braiding operations with stationary anyons of the same type ordered canonically on the line of the walk. Abelian as well as non-Abelian anyons are studied and it is shown that they have very different properties. Abelian anyonic walks demonstrate the expected quadratic quantum speedup. Non-Abelian anyonic walks are much more subtle. The exponential increase of the system's Hilbert space and the particular statistical evolution of non-Abelian anyons give a variety of new behaviors. The position distribution of the walker is related to Jones polynomials, topological invariants of the links created by the anyonic world-lines during the walk. Several examples such as the $\mathrm{SU}(2)_{k}$ and the quantum double models are considered that provide insight to the rich diffusion properties of anyons.
\end{abstract}

PACS numbers: 05.30.Pr, 05.40.Fb

\section{INTRODUCTION}

Quantum versions of random walks came to prominence in quantum information theory through the search for efficient new quantum algorithms. Since classical random walks provide the techniques for some of the best classical algorithms, it was natural to look for a quantum equivalent. Typically, a quantum walk gives a quadratic algorithmic speed up over classical ones [1] and for some problems an exponential speed up is possible [2]. This is a generalization of the faster spreading behavior shown by the simplest quantum walk, a single walker on an infinite line.

Quantum walks have since found wider applications than their algorithmic origins. In essence a quantum walk is a discrimination of a diffusion process. For example, they has been employed in modeling transport of charge or energy in biological systems [3], in physical systems to show delocalization and quantum coherence [4, 5, 6], as a model system interpolating between quantum and classical behavior [7], as well as a model amenable to quantum simulation of its asymptotics [8]. On the algorithmic side they have been proved to be universal for quantum computation [9, 10] and they have been physically implemented, both as a computation on a quantum computer [11] and a physical walk (e.g. [12, 13]). Quantum walks have been studied theoretically on many different structures: in higher dimensions [14], under the effects of decoherence [15], with Dirac spin particles [16], and with a huge range of variations on the basic walk dynamics. For accessible introductions and reviews, see, for example, [17, 18, 19].

Here we focus on the question of how the quantum walk distribution can be affected by the statistical properties of the walker. Non-interacting bosons in quantum walks have effectively already been shown to have identical properties to single quantum walkers through experiments with coherent light [7, 12]. Omar et al. [20] studied quantum walks with two bosonic or fermionic walkers. Entanglement in the initial state of these walkers gives a strikingly different behavior with respect to the standard quantum walk.

More exotic statistics than the bosonic or the fermionic can be found in two-dimensional systems where anyons can appear. Exchanging two anyons can introduce phase factors or unitary transformations. These evolutions are different representations of the braiding group. Envisioning how quantum walks can be performed with anyons one quickly realizes that the problem takes the form of diffusion of a walker-anyon in the presence of other regularly arranged static anyons. In particular, we embed a one dimensional quantum walker into a two dimensional medium with a 
canonical line ordering of the anyons from left to right. When the anyonic walker is moving among the static anyons then anyonic braiding is realized evolving the overall state of the system in a non-trivial way. Hence, by considering an anyonic walk we enrich the one particle diffusion problem with statistical properties.

Anyonic quantum walks is not only an academic curiosity, but it can be considered as a quantum simulator modeling the diffusion of extended objects. In order to keep track of the statistical properties of anyons it is useful to visualize their world-lines that encode their braiding history. When the walker moves from its initial position it braids with the straight world-lines of the static anyons. Finding the walker at a certain position after a number of steps requires the consideration of all the different paths of the same length with the same initial and final positions. This gives rise to world-line links the complexity of which increases fast with respect to the number of the walker steps. The diffusion of extended objects has applications in biophysics and polymer physics [21]. Here, we are interested in the quantum version of such diffusion processes.

Before turning to the anyonic quantum walks we briefly introduce the standard quantum walk as well as the anyons and their properties. In Sec. IW we present in detail the general formalism of the anyonic quantum walk. In Sec. III we demonstrate that Abelian anyonic quantum walks have similar asymptotic behavior as the standard quantum walks. In Sec. IV we consider the non-Abelian anyonic quantum walks and we express the walker probability distribution in terms of the Kauffman brackets. Several examples are explicitly solved for a small number of walker steps that indicate a wide range of diffusion properties ranging between the classical and the quantum walks. Finally, in Sec. V we present our conclusions and we point out interesting future directions.

\section{A. Quantum walk on a line}

Quantum walks have been studied in both the continuous time [22] and discrete time [23, 24] versions. We briefly describe the discrete time quantum walk on the infinite line, which will be later generalized to the anyonic case. It is defined in direct analogy with a classical random walk: there is a walker carrying a coin which is tossed each time step and the walker moves left or right according to the heads or tails outcome of the coin toss.

We denote the basis states for the quantum walk as an ordered pair of labels in a "ket" $|s\rangle_{\text {space }} \otimes|j\rangle_{\text {spin }}$, where $s \in \mathbb{Z}$ is the position and $j \in\{0,1\}$ is the spin-like state of the coin. A unitary coin operator is used at each time step and then a shift operation is applied to move the walker to its new positions. The simplest coin toss is the Hadamard operator $H$, defined by its action on the basis states $|s, j\rangle$ as

$$
\begin{aligned}
& H|s, 0\rangle=\frac{1}{\sqrt{2}}(|s, 0\rangle+|s, 1\rangle) \\
& H|s, 1\rangle=\frac{1}{\sqrt{2}}(|s, 0\rangle-|s, 1\rangle),
\end{aligned}
$$

and the shift operation $T$ acts on the basis states as

$$
\begin{aligned}
& T|s, 0\rangle=|s-1,0\rangle \\
& T|s, 1\rangle=|s+1,0\rangle .
\end{aligned}
$$

A single step of the quantum walk consists of $W=T H$ applied to the quantum walker plus spin. After performing $t$ steps of the quantum walk, where the walker is initially in state $|\Phi(0)\rangle$, we obtain the final state

$$
|\Phi(t)\rangle=W^{t}|\Phi(0)\rangle \text {. }
$$

Ultimately we are interested in the probability distribution $P(s, t)$ of the spatial location, $s$, of the walker at time $t$ that is given by

$$
(P(1, t), P(2, t), \ldots)=\operatorname{diag}\left(\operatorname{tr}_{\text {spin }}[|\Phi(t)\rangle\langle\Phi(t)|]\right)
$$

As the walk progresses, quantum interference occurs whenever there is more than one possible path of $t$ steps that leads to the same position. This interference is both constructive and destructive, which causes some probabilities to 
be amplified or decreased at each timestep. This leads to the different behavior compared to its classical counterpart. In the latter the position of a walker, following a classical random walk on a line, spreads out in a binomial distribution about its starting point. In Refs. [24], Ambainis et al. proved that the quantum walk on a line spreads in $O\left(t^{2}\right)$ compared to a classical random walk which spreads in $O(t)$, where the spreading is being measured as the second moment about a single initial starting point.

\section{B. Anyons and their properties}

It is commonly accepted that point-like particles, elementary or not, come in two species, bosons or fermions. This statistical label is determined by the behavior of their wave function when two identical non-interacting particles are exchanged. For bosons the wave function is unchanged while for fermions it acquires a phase of $\pi$, i.e., changes sign. These are the only observed statistical behaviors for particles that exist in our three dimensional world. If one restricts to two dimensions there are more possibilities in the particle statistics. In this case, when two particles are exchanged their wave function can be evolved by an arbitrary phase factor or even a unitary operator that creates superpositions in an internal space of the particles. These particles, named anyons by Frank Wilczek, can appear as effective quasiparticles, such as vortices, in two dimensional many body systems. They are manifested in the Fractional Quantum Hall Effect (FQHE) [25, 26] and they are expected to appear in p-wave superconductors [27, 28], topological insulators [29, 30] and a variety of lattice models [31, 32, 33, 34, 35].

Anyons can be created from the vacuum in pairs, they can be braided around each other and they can be fused together. While the pair creation results in a particle and its antiparticle much in the same way as usual particles, braiding operations and fusion of anyons are rather unique. This is due to the non-local degrees of freedom carried between anyons that give rise to their exotic statistics. One can think of it like an internal 'spin'. When two anyons are braided then their internal 'spin' state either acquires a phase factor, $e^{i \phi}$ (Abelian anyons) or it gets evolved by a unitary matrix, $b$ (non-Abelian anyons). The fusion corresponds to the tensor product of these 'spins' with a restriction on the maximum possible 'spin' value. When two anyons, $a$ and $b$, are fused then more than one outcomes are possible depending on their internal state. Symbolically we have $a \times b=c+d+\ldots$
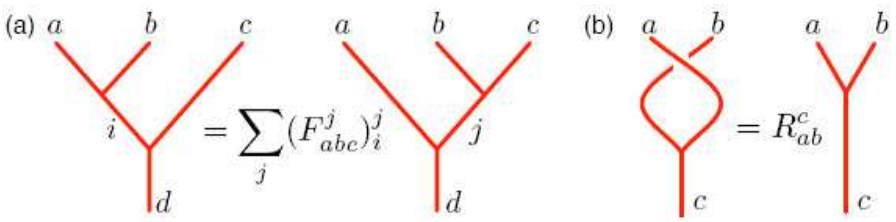

FIG. 1: The (a) $F$ and (b) $R$ moves dictated from the fusion and braiding properties of anyons.

Three anyons $a, b$ and $c$ can be fused to a final anyon $d$ in two different ways. Either one can fuse $a$ with $b$ and its result $i$ with $c$ or one can fuse $b$ with $c$ and its result $j$ with $a$. These two different processes correspond to two different bases of the collective state of the anyons. The different ways of combining particles $a, b, c$ to yield $d$ is given by the recoupling formula: $|(a b) c \rightarrow d ; x\rangle=\sum_{x^{\prime}}\left(F_{a b c}^{d}\right)_{x}^{x^{\prime}} \mid\left(a(b c) \rightarrow d ; x^{\prime}\right\rangle$ as seen in Fig. 11 a). The braiding of two anyons $a, b$ with fusion channel $c$ is described by $R_{a b}^{c}$ as seen in Fig. 1(b). To obtain the braiding result of two anyons that do not have a direct fusion channel (see for example anyons $b$ and $c$ on the left hand side of Fig.11a)) one can use the $F$ matrix to obtain their braiding element, $F^{-1} R F$. Anyons also carry a quantum dimension $d$. This accounts for the dimension of the internal space of the anyons and provides the scaling of the total Hilbert space. For example, $n$ anyons with quantum dimension $d$ have a Hilbert space with dimension $d^{n}$. Abelian anyons have $d=1$ and non-Abelian ones have necessarily $d>1$. Note that, in contrast to usual spin, $d$ can be an irrational number. For more details on anyons and on topological quantum computation see, e.g. [36] and references therein. 


\section{WALKING ANYONS}

In order to reveal the statistics of anyons in a quantum walk, we consider a single anyonic walker braiding around others in fixed positions. The anyons involved are all of the same type $\sigma$. The walker has an attached die degree of freedom which gives it distinguishability. However this distinguishability does not negate the action of braiding on the non-local statistical degrees of the system. The anyons are placed in a canonical order in the plane with position labelled by an integer $s$, as seen in Fig. 2(a). For an anyon at position $s$, other anyons with positions $s^{\prime}<s\left(s^{\prime}>s\right)$ will be said to be on the left (right). We restrict to $n \bmod 4=2 \times$ odd in order to have an odd number of pairs such that the initial state with the marked anyonic walker and its partner located at the middle will have an equal number of pairs to their right and left. This facilitates the construction, if desired, of a symmetric evolution.

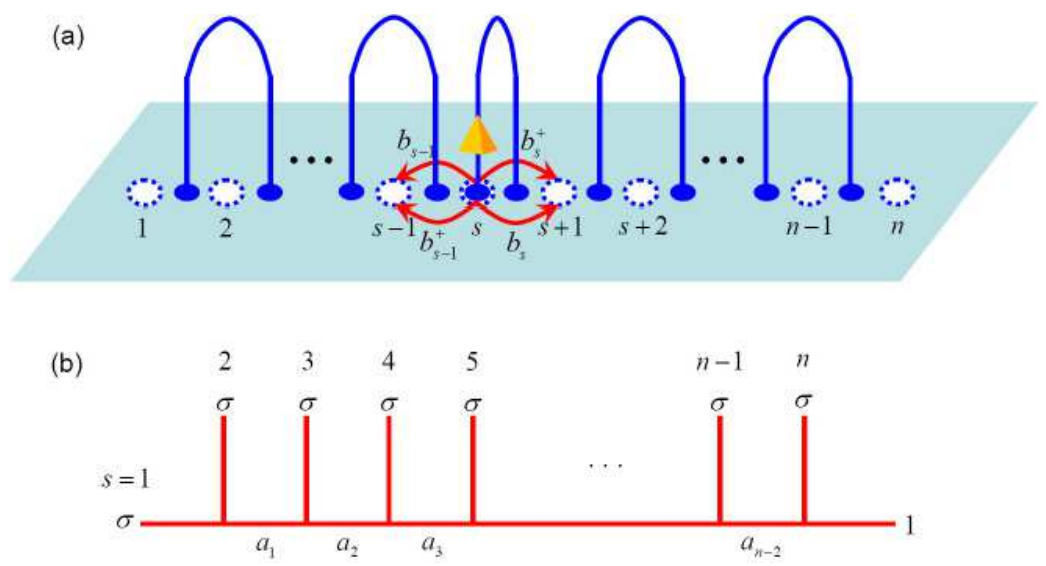

FIG. 2: The anyonic quantum walk. (a) A quantum walk of the anyonic walker with a four state die attached. It walks along one of four braids depending on the state of the die on sites labelled by $s=1$ to $s=n$ (white circles) positioned in between stationary anyons (blue circles). (b) A basis state $\left|\Psi\left(a_{1}, a_{2}, \ldots, a_{n-2}\right)\right\rangle$ of the non-local fusion space representing the sequential topological charge measurements of $n$ type $\sigma$ anyons with total charge zero (vacuum, also denoted as 1 ).

Basis states are labelled by $\left|\Psi\left(a_{1}, a_{2}, \ldots a_{n-2}\right)\right\rangle$ according to the fusion outcomes as indicated by Fig. 2(b). The state with nearest neighbor vacuum pairs depicted in Fig. 2(a) is

$$
\left|\Psi_{0}\right\rangle=|\Psi(1, \sigma, 1, \sigma, \ldots, 1, \sigma)\rangle
$$

where 1 is the vacuum particle, as can be verified by considering the consistent fusion ordering. If desired one could modify the protocol to give a symmetric distribution for the walker by changing the initial state to provide for a symmetric spatial distribution for the anyons. Taking the vacuum partner of the walker and winding counterclockwise halfway around all its neighbors, the initial fusion state $\left|\Psi_{0}\right\rangle=b_{n} b_{n-1} \cdots b_{(n+3) / 2}|\Psi(1, \sigma, 1, \sigma, \ldots, 1, \sigma)\rangle$ provides for the walker anyon to have the same number of vacuum pairs on its left and right.

The Hilbert space of our system is

$$
\mathcal{H}(n)=\mathcal{H}_{\text {anyons }}(n) \otimes \mathcal{H}_{\text {die }}(n)
$$

The die contains both a discrete spatial index indicating its position at the location of the anyons on the plane and 4 internal spin states: $\mathcal{H}_{\text {die }}(n)=\mathcal{H}_{\text {space }}(n) \otimes \mathcal{H}_{\text {spin }}$, where $\mathcal{H}_{\text {space }}=\operatorname{span}_{\mathbb{C}}\{|s\rangle\}_{s=1}^{n}$ and $\mathcal{H}_{\text {spin }}=\operatorname{span}_{\mathbb{C}}\{|j\rangle\}_{j=0}^{3} . \operatorname{The}^{3}$ anyonic Hilbert space is further decomposed as $\mathcal{H}_{\text {anyons }}(n)=\mathcal{H}_{\text {local }}(n) \otimes \mathcal{H}_{\text {fusion }}(n)$ the first factor describing degrees of freedom local to each anyon and the later factor the non-local or fusion degrees of freedom. We restrict to a contractible surface so that the homological degrees of freedom are trivial. Furthermore, the local degrees of freedom, while measurable, will be conserved during this walk and will be ignored.

Fusion degrees of freedom are determined by the fusion rules $a_{i} \times a_{j}=\sum_{k} N_{a_{i} a_{j}}^{a_{k}} a_{k}$, where $N_{a b}^{c} \in \mathbb{N}$ counts the number of ways to combine anyons of type $a$ and $b$ to obtain $c$. The corresponding Hilbert state space 
of $n \sigma$ anyons with total charge zero is $\mathcal{H}_{\text {fusion }}(n)=\operatorname{span}_{\mathbb{C}}\left\{\left|\Psi\left(\left\{a_{j}\right\}_{j=1}^{n-2}\right)\right\rangle\right\}$ with dimension $\operatorname{dim}\left(\mathcal{H}_{\text {fusion }}(n)\right)=$ $\sum_{a_{1}, a_{2}, \ldots, a_{n-2}} N_{\sigma \sigma}^{a_{1}} N_{a_{1} \sigma}^{a_{2}} N_{a_{2} \sigma}^{a_{3}} \cdots N_{a_{n-2} \sigma}^{1}$. We will restrict to cases where the fusion spaces are one dimensional, i.e. $N_{a b}^{c}<2$. Braiding relations are determined by the $F$ and $R$ matrices. The action of the generators $\left\{b_{s}\right\}_{s=1}^{n-1}$ of the braid group $\mathcal{B}_{n}$ on $\mathcal{H}_{\text {fusion }}(n)$ is given by the amplitudes

$$
\left\langle\Psi\left(\left\{a_{j}^{\prime}\right\}\right)\left|b_{k}\right| \Psi\left(\left\{a_{j}\right\}\right)\right\rangle=\left\{\begin{array}{lc}
\prod_{m=k}^{n-2} \delta_{a_{m}^{\prime}, a_{m}} R_{\sigma \sigma}^{a_{1}}, & k=1 \\
\prod_{m=k}^{n-2} \delta_{a_{m}^{\prime}, a_{m}} \sum_{x}\left(\left[F_{\sigma \sigma \sigma}^{a_{k}}\right]^{-1}\right)_{x}^{a_{k-1}^{\prime}} R_{\sigma \sigma}^{x}\left(F_{\sigma \sigma \sigma}^{a_{k}}\right)_{a_{k-1}}^{x}, & k=2 \\
\prod_{\ell=1}^{k-2} \delta_{a_{\ell}^{\prime}, a_{\ell}} \prod_{m=k}^{n-2} \delta_{a_{m}^{\prime}, a_{m}} \sum_{x}\left(\left[F_{a_{k-2} \sigma \sigma}^{a_{k}}\right]^{-1}\right)_{x}^{a_{k-1}^{\prime}} R_{\sigma \sigma}^{x}\left(F_{a_{k-2} \sigma \sigma}^{a_{k}}\right)_{a_{k-1}}^{x}, & 2<k<n-1 \\
\prod_{m=k}^{n-2} \delta_{a_{m}^{\prime}, a_{m}} R_{\sigma \sigma}^{a_{n-3},} & k=n-1
\end{array}\right.
$$

where the formula $b=F^{-1} R F$ is employed when the walker does not have direct fusion channels with the rest of the anyons.

The initial state of our system is

$$
|\Phi(0)\rangle=\left|\Psi_{0}\right\rangle_{\text {fusion }}\left|s_{0}=\frac{n}{2}\right\rangle_{\text {space }}|\psi\rangle_{\text {spin }}
$$

The quantum walk algorithm consists of an iterative sequence of operations $W=T U$ where $U$ is the die tossing operation that acts trivially on the position of the walker and $T$ is the conditional braiding operator. We can introduce a virtual tensor product structure to the spin states: $|0\rangle_{\text {spin }}=|0\rangle_{\mathrm{x}}|0\rangle_{\mathrm{y}},|1\rangle_{\text {spin }}=|0\rangle_{\mathrm{x}}|1\rangle_{\mathrm{y}},|2\rangle_{\text {spin }}=|1\rangle_{\mathrm{x}}|0\rangle_{\mathrm{y}},|3\rangle_{\text {spin }}=$ $|1\rangle_{\mathrm{x}}|1\rangle_{\mathrm{y}}$ where $|0\rangle_{\mathrm{y}}\left(|1\rangle_{\mathrm{y}}\right)$ are modes which are behind (in front of) their neighbors, and $|0\rangle_{\mathrm{x}}\left(|1\rangle_{\mathrm{x}}\right)$ are modes moving left or right. For the die toss we pick (in the product basis)

$$
U=\frac{1}{\sqrt{2}}\left(\begin{array}{cc}
1 & i \\
i & 1
\end{array}\right) \otimes \frac{1}{\sqrt{2}}\left(\begin{array}{cc}
1 & i \\
i & 1
\end{array}\right)=e^{i \frac{\pi}{4}\left(X_{x}+X_{y}\right)},
$$

which can be interpreted as a pair of beam splitters on the two axes. Each component of $U$ is a symmetrized version of the Hadamard operation of Eqn. (11). Of course a continuum of tossing operations could be considered but this decomposition into a virtual tensor product has advantages as described below. The conditional braiding operation $T$ has the following action

$$
T|\Psi\rangle_{\text {fusion }}|s\rangle_{\text {space }}|\kappa\rangle_{\mathrm{x}}|\gamma\rangle_{\mathrm{y}}=b_{s+\kappa-1}^{(-1)^{\mathrm{\kappa} \oplus 2 \gamma}}|\Psi\rangle_{\text {fusion }}|s+2 \kappa-1\rangle_{\text {space }}|\kappa\rangle_{\mathrm{x}}|\gamma\rangle_{\mathrm{y}}
$$

over the spatial range $0<s<n+1$.

It is convenient to introduce the unnormalized spinor

$$
\left.|\vec{\Phi}(s, t)\rangle={ }_{\text {spin }}\langle 0 \mid \Phi(s, t)\rangle, \text { spin }\langle 1 \mid \Phi(s, t)\rangle, \text { spin }\langle 2 \mid \Phi(s, t)\rangle \text {,spin }\langle 3 \mid \Phi(s, t)\rangle\right)^{T},
$$

so that an arbitrary state at time $t$ is

$$
|\Phi(t)\rangle=\sum_{s=1}^{n+1}|\vec{\Phi}(s, t)\rangle .
$$

In order to avoid boundary conditions on the walk we study the behavior for $t<n / 2$. 


\section{ABELIAN ANYONIC QUANTUM WALK}

\section{A. Variance of Abelian anyonic walks}

Employing the method of Brun et al. [37, 38, 39] one can analytically evaluate the variance of the distributions corresponding to the Abelian anyonic walks by determining the moments of the resulting distribution. For simplicity we relabel the positions so that the initial walker position in the middle of the anyonic chain is given by $s_{0}=0$, i.e. $|\Phi(0)\rangle=|s=0\rangle_{\text {space }} \otimes|\psi\rangle_{\text {spin. }}$. In Fourier components we have

$$
|s=0\rangle=\int_{-\pi}^{\pi} \frac{d k}{2 \pi}|k\rangle
$$

In the Fourier transformed basis the evolution operator $W$ acts as $W(|k\rangle \otimes|\psi\rangle)=|k\rangle \otimes M_{k}|\psi\rangle$ so after $t$ steps the state evolves to

$$
W^{t}|\Phi(0)\rangle=\int_{-\pi}^{\pi} \frac{d k}{2 \pi}|k\rangle \otimes\left(M_{k}\right)^{t}|\psi\rangle
$$

One can write a closed form for the moments of the resulting distribution. By employing the expansion of the identity in the position $s$ basis, $\mathbb{1 1}=\sum_{s}|s\rangle\langle s|$ and $\langle s \mid k\rangle=e^{-i k s}$ we obtain

$$
\begin{aligned}
\left\langle s^{m}\right\rangle_{t} & =\frac{1}{(2 \pi)^{2}} \sum_{s} s^{m} \int d k \int d k^{\prime} e^{-i s\left(k-k^{\prime}\right)}\left\langle\Phi(0)\left|\left(M_{k}^{\dagger}\right)^{t}\left(M_{k^{\prime}}\right)^{t}\right| \Phi(0)\right\rangle \\
& =\frac{i^{m}}{2 \pi} \int d k \int d k^{\prime} \delta^{(m)}\left(k-k^{\prime}\right)\left\langle\Phi(0)\left|\left(M_{k}^{\dagger}\right)^{t}\left(M_{k^{\prime}}\right)^{t}\right| \Phi(0)\right\rangle .
\end{aligned}
$$

Now we can integrate by parts the derivatives that act on the $\delta$-function. For the case of $m=1$ we obtain

$$
\langle s\rangle_{t}=\frac{i}{2 \pi} \int d k\left\langle\Phi(0)\left|\left(M_{k}^{\dagger}\right)^{t} \frac{d\left(M_{k}\right)^{t}}{d k}\right| \Phi(0)\right\rangle .
$$

We have that $d M_{k} / d k=-i\left(P_{R}-P_{L}\right) M_{k}$, where $P_{R, L}$ are the projector operators for moving the walker right or left with $P_{R}+P_{L}=1$. Finally, we obtain

$$
\langle s\rangle_{t}=\frac{1}{2 \pi} \sum_{j=1}^{t} \int d k\left\langle\Phi(0)\left|\left(M_{k}^{\dagger}\right)^{j}\left(P_{R}-P_{L}\right)\left(M_{k}\right)^{j}\right| \Phi(0)\right\rangle .
$$

Similarly for $m=2$ we have

$$
\left\langle s^{2}\right\rangle_{t}=\frac{1}{2 \pi} \sum_{j=1}^{t} \sum_{j^{\prime}=1}^{t} \int d k\left\langle\Phi(0)\left|\left(M_{k}^{\dagger}\right)^{j}\left(P_{R}-P_{L}\right)\left(M_{k}\right)^{j-j^{\prime}}\left(P_{R}-P_{L}\right)\left(M_{k}\right)^{j^{\prime}}\right| \Phi(0)\right\rangle .
$$

One can further employ the eigenstates, $\left|\lambda_{l}(k)\right\rangle$, and eigenvalues, $\lambda_{l}(k)$, of $M_{k}(l$ runs through the eigenstates) to explicitly evaluate the moments. Let us expand the initial state in this basis $|\Phi(0)\rangle=\sum_{l} c_{l}(k)\left|\lambda_{l}(k)\right\rangle$. After $t$ steps the state becomes $\left(M_{k}\right)^{t}|\Phi(0)\rangle=\sum_{l} e^{i \lambda_{l}(k) t} c_{l}(k)\left|\lambda_{l}(k)\right\rangle$. With this in mind the first moment becomes

$$
\langle s\rangle_{t}=t-\frac{1}{\pi} \int d k \sum_{l, l^{\prime}} c_{l}^{*}(k) c_{l^{\prime}}(k)\left\langle\lambda_{l}(k)\left|P_{L}\right| \lambda_{l^{\prime}}(k)\right\rangle \sum_{j=1}^{t} e^{i\left[\lambda_{l^{\prime}}(k)-\lambda_{l}(k)\right] j} .
$$

If the matrix $M_{k}$ is non-degenerate then most of the terms in $\langle s\rangle_{t}$ will be oscillatory and they will average to zero over time. The non-zero contributions comes from the diagonal terms

$$
\langle s\rangle_{t}=\left(1-\frac{1}{\pi} \int d k \sum_{l}\left|c_{l}(k)\right|^{2}\left\langle\lambda_{l}(k)\left|P_{L}\right| \lambda_{l}(k)\right\rangle\right) t+\text { Oscillatory terms. }
$$


Similarly for the second moment we have

$$
\left\langle s^{2}\right\rangle_{t}=\left(1-\frac{2}{\pi} \int d k \sum_{l}\left|c_{l}(k)\right|^{2}\left\langle\lambda_{l}(k)\left|P_{L}\right| \lambda_{l}(k)\right\rangle\left\langle\lambda_{l}(k)\left|P_{R}\right| \lambda_{l}(k)\right\rangle\right) t^{2}+\text { Oscillatory terms and lower } t \text { orders. }
$$

For the Abelian anyonic walker,

$$
M_{k}=e^{i k} M_{+}+e^{-i k} M_{-}=e^{-i k Z_{x}} e^{i \phi Z_{x} \otimes Z_{y}} e^{i \frac{\pi}{4}\left(X_{x}+X_{y}\right)} .
$$

The eigenvalues of $M_{k}$ come in conjugate pairs

$$
\lambda_{1}(k)=e^{-i \beta_{-}(k)}, \quad \lambda_{2}(k)=e^{i \beta_{-}(k)} \quad \lambda_{3}(k)=e^{-i \beta_{+}(k)}, \quad \lambda_{4}(k)=e^{i \beta_{+}(k)},
$$

where $\beta_{ \pm}(k)=\cos ^{-1}\left[\frac{1}{2}\left(\cos (k) \cos (\phi) \pm \sqrt{\left(\cos ^{2}(\phi)-2\right) \cos ^{2}(k)+2}\right)\right]$.

The eigenvectors are

$$
\begin{aligned}
&\left|\lambda_{1}(k)\right\rangle=\left|v\left(\beta_{-}\right)\right\rangle,\left|\lambda_{2}(k)\right\rangle=-i Y \otimes Z \overline{\left.\lambda_{1}(k)\right\rangle}, \\
&\left|\lambda_{3}(k)\right\rangle=\left|v\left(\beta_{+}\right)\right\rangle, \quad\left|\lambda_{4}(k)\right\rangle=-i Y \otimes Z \overline{\left|\lambda_{3}(k)\right\rangle},
\end{aligned}
$$

where

$$
\begin{aligned}
|v(\beta)\rangle=\frac{1}{\sqrt{\mathcal{N}}} & {\left[\left(e^{-2 i(k+\beta)}+1+e^{-2 i \beta}-e^{i(-k+\phi-\beta)}+e^{2 i(\phi-\beta)}-2 e^{i(-k+\phi-3 \beta)}-e^{i(k+\phi-\beta)}\right)|0\rangle_{x}|0\rangle_{y}\right.} \\
& +i\left(e^{-i(\phi+k+\beta)}-e^{-2 i(k+\beta)}-1+e^{-i(k-\phi+\beta)}\right)|0\rangle_{x}|1\rangle_{y} \\
& \left.+i\left(e^{-i(\phi+k+\beta)}-1-e^{-2 i \beta}+e^{i(k+\phi-\beta)}\right)|1\rangle_{x}|0\rangle_{y}+\left(e^{2 i(\phi-\beta)}-1\right)|1\rangle_{x}|1\rangle_{y}\right],
\end{aligned}
$$

with $1 / \sqrt{\mathcal{N}}$ the normalization factor. Employing the eigenvectors and eigenvalues of $M_{k}$ and using Eqs. (6), (7) the variance, $v=\left\langle s^{2}\right\rangle-\langle s\rangle^{2}$, can be evaluated, as seen in Fig. 3.

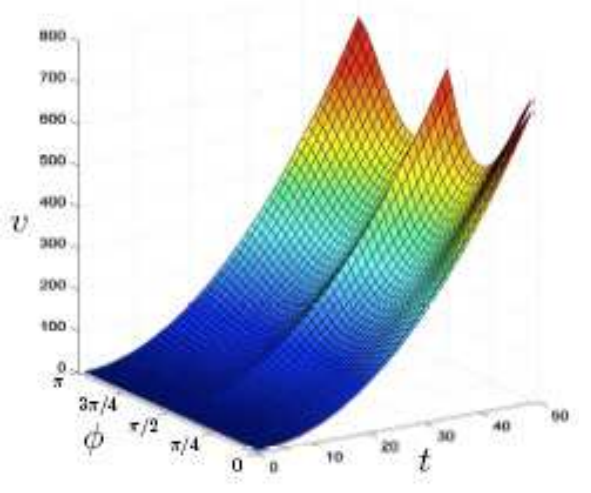

FIG. 3: The variance, $v=\left\langle s^{2}\right\rangle-\langle s\rangle^{2}$, of the Abelian quantum walk as a function of the number of steps, $t$, and the statistical angle, $\phi$. The two plots shown are the analytical results (bottom surface), based on Eqn. (8) and (9), and the numerical ones (top surface) giving almost identical results. A quadratic behavior of the variance is witnessed as expected from the standard quantum case.

\section{B. Results}

It is rather straightforward to perform a numerical simulation of the Abelian quantum walk on the line for relatively large number of steps. This gives the exact distribution of the walker and one can obtain the corresponding variance, 
$v=\left\langle s^{2}\right\rangle-\langle s\rangle^{2}$, as a function of the number of steps, $t$, and the statistical angle, $\phi$, of the anyons. The numerically obtained variance and the analytical results of the previous subsection are depicted in Fig. 3, appearing to be almost identical, with a small difference due to the neglect of terms in the analytic calculations. We observe the expected quadratic behavior that corresponds to the standard quantum case. There is a weak dependence on the anyonic statistical angle $\phi$. This is due to the form of the coin evolution that includes the term $e^{i \phi Z_{y} \otimes Z_{x}}$ (see Eq. (10)). For the case where $\phi$ is a multiple of $\pi / 2$ this term has a tensor product structure that splits the system into the tensor product of two quantum walks, each one with a two dimensional coin. Thus, we expect to obtain the same variance as the standard quantum walk with a single two dimensional coin. Note that these cases include the quantum walks of bosons $(\phi=0)$ and fermions $(\phi=\pi)$. For generic values of $\phi$ the walk entangles the two coins. As a result the interference effect that gives the speed up of the quantum evolution gets diluted over the four dimensional space of the two coins and the slope of the variance is decreased. This is in agreement with previous studies, where quantum walks with multiple coins were considered [37, 38].

\section{NON-ABELIAN ANYONIC QUANTUM WALK}

In this section we would like to perform a quantum walk with non-Abelian anyons. For simplicity we slightly change the quantum walk. Now the walker moves to the left and to the right by always braiding in the anti-clockwise sense with its neighboring anyons. This can be achieved by employing only one coin (which has two degrees of freedom). One can show that for a given non-Abelian anyonic model the braidwords that result from this quantum walk have the same computational power as arbitrary braidwords. Initially, note that having static $n$ anyons and braiding one moving anyon clockwise or anticlockwise around them can realize the full computational power of the anyonic model [40]. Restricting only to anti-clockwise braidings does not restrict the possible obtained unitaries. Indeed, for the finite braiding groups we consider here one can show that there exists an integer $k$ such that for a generic braid element $b_{i}$ it is $b_{i}^{k}=1 \Rightarrow b_{i}^{k-1}=b_{i}^{-1}$ (see [41]). Thus, braidings equivalent to clockwise moves can be produces given enough anti-clockwise ones. Notably, fixing the chirality of the braiding means that Abelian sectors of any physical theory will not contribute to the walker's distribution since all walking paths accumulate the same overall phase. Hence the single coin protocol probes the truly non-Abelian statistics.

Consider $t$ steps of the quantum walker and, for brevity, define

$$
B_{\vec{a}}^{t} \equiv \prod_{r=0}^{t-3} b_{s_{t}+2\left(a_{1}+\ldots a_{t-r}\right)-(t-r)} b_{s_{t}+2 a_{1}+a_{2}-2} b_{s_{t}+a_{1}-1}
$$

The evolution of the system can be given by the unitary operator

$$
V^{t}=\sum_{s_{t} \in[t+1, n-t+1]} \sum_{\vec{a} \in\{0,1\}} \prod_{r=1}^{t} P_{a_{r}} U \otimes B_{\vec{a}}^{t} \otimes\left|s_{t}+2\left(a_{1}+\ldots+a_{t}\right)-t\right\rangle\left\langle s_{t}\right| .
$$

Starting from the walker in position $m$ and the coin qubit in state $|\psi\rangle$ one obtains after $t$ steps the state

$$
|\Psi(t)\rangle=V^{t}|\psi\rangle|\alpha\rangle|m\rangle=\sum_{\vec{a}}\left(\prod_{r=1}^{t} P_{a_{r}} U\right)|\psi\rangle B_{\vec{a}}^{t}|\alpha\rangle\left|\bar{n}_{\vec{a}}^{t}\right\rangle
$$

where $\vec{a}$ is a $t$ dimensional vector with $r$-th component 0 or 1 depending if the walker is moving, respectively, left or right at the $r$-th step, $\bar{n}_{\vec{a}}^{t}=m+2\left(a_{1}+\ldots+a_{t}\right)-t$ and the initial fusion state of the anyons is $|\alpha\rangle=|0101 \ldots 01\rangle$ as in [42]. One can write the corresponding density matrix $\rho^{t}=|\Psi(t)\rangle\langle\Psi(t)|$ as

$$
\rho(t)=\sum_{\vec{a}, \vec{a}^{\prime}}\left(\prod_{r=1}^{t} P_{a_{r}} U\right)|\psi\rangle\left\langle\psi\left|\left(\prod_{r=1}^{t} P_{a_{r}^{\prime}} U\right) B_{\vec{a}}^{t}\right| \alpha\right\rangle\left\langle\alpha\left|B_{\vec{a}^{\prime}}^{t}\right| \bar{n}_{\vec{a}}^{t}\right\rangle\left\langle\bar{n}_{\vec{a}^{\prime}}^{t}\right| .
$$


Importantly, the coin, walker position and anyonic Hilbert spaces can be traced out independently. For brevity let us set

$$
\mathcal{U}_{\vec{a} \vec{a}^{\prime}}^{t}=\left(\prod_{r=1}^{t} P_{a_{r}} U\right)|\psi\rangle\langle\psi|\left(\prod_{r=1}^{t} P_{a_{r}^{\prime}} U\right)
$$

and

$$
\mathcal{Y}_{\vec{a} \vec{a}^{\prime}}^{t}=B_{\vec{a}}^{t}|\alpha\rangle\langle\alpha| B_{\vec{a}^{\prime}}^{t \dagger}
$$

We would like to obtain the position distribution of the walker regardless of the coin and anyonic states. For that we need to trace the coin states and the fusion space of the anyons resulting in

$$
\operatorname{tr}_{\text {coin }} \operatorname{tr}_{\text {anyon }} \rho(t)=\sum_{\vec{a}, \vec{a}^{\prime}} \operatorname{tr} \mathcal{U}_{\vec{a} \vec{a}^{\prime}}^{t} \operatorname{tr} \mathcal{Y}_{\vec{a} \vec{a}^{\prime}}^{t}\left|\bar{n}_{\vec{a}}^{t}\right\rangle\left\langle\bar{n}_{\vec{a}^{\prime}}^{t}\right| .
$$

Due to the choice of coin manipulations one needs to have paths $\vec{a}$ and $\vec{a}^{\prime}$ that end up at the same coin state for the trace to be non-trivial. To simplify the presentation we consider the composite loop $\vec{a} \vec{a}^{\prime}$ corresponding to paths $\vec{a}$ and $\vec{a}^{\prime}$ that start at the same point and end at the same point after the same number of steps. Thus, they are contributing to the diagonal part of $\operatorname{tr}_{\text {coin }} \operatorname{tr}_{\text {anyon }} \rho(t)$.

For a Hadamard coin flip operation $U=H$ (see Eq. (1)) one can easily evaluate that

$$
\operatorname{tr} \mathcal{U} \mathcal{a}_{\vec{a} \vec{a}^{\prime}}^{t}=\frac{1}{2^{n}}(-1)^{z},
$$

where $z$ is the number of all successive pairs of 1's in the loop $\vec{a} \vec{a}^{\prime}$. Hence, successive zeros and ones in the loop $\vec{a} \vec{a}^{\prime}$ can be compressed. While the coin trace is rather straightforward care needs to be taken when one takes the trace over the anyonic degrees of freedom. It can be shown [42] that the trace of unitary representations of the braiding group corresponds to the Markov trace of the braid graphs described above. As initial condition we adopt the anyons to be created pairwise from the vacuum. Hence, the trace of the braidings gives the Kauffman bracket of the Plat traced braidings.
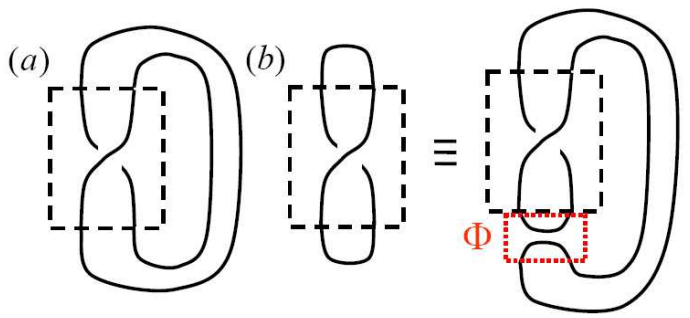

FIG. 4: The Markov (a) and Plat (b) tracing of a braid. In (b) the equivalence of the Plat and the Markov traces is depicted, which is established with the help of the Temperley-Lieb algebra element $\Phi$ [42] that has a simple diagrammatic representation.

In [42] it has been shown that $|\alpha\rangle\langle\alpha|=\Phi_{1} \Phi_{3} \ldots \Phi_{n-1} / d^{n / 2}$. Intuitively, this means that the density matrix of the initial anyonic state, given by the pair creation of particles from the vacuum, corresponds to the product of TemperleyLieb algebra elements $\Phi_{i}$ as depicted in Fig. 4. Thus one has

$$
\operatorname{tr}\left(B_{\vec{a}}^{t}|\alpha\rangle\langle\alpha| B_{\vec{a}^{\prime}}^{t \dagger}\right)=\frac{1}{d^{n / 2-1}}\left\langle\left(B_{\vec{a}^{\prime}}^{t} B_{\vec{a}}^{t}\right)^{\text {Plat }}\right\rangle
$$

where $B_{\overrightarrow{\vec{A}^{\prime}}}^{\dagger} B_{\vec{a}}$ have been Plat traced and then the corresponding Kauffman bracket has been evaluated. The Kauffman bracket [43] of a link $\langle L\rangle(A)$ is a Laurent polynomial in the complex variable $A$ and can be computed by smoothing each crossing according to the Skein relation shown in Fig. 5. Up to a phase factor they are equal to the Jones polynomials [44], topological invariants that facilitate the distinction between inequivalent links. Kauffman brackets have the following property, $\langle L \mathrm{O}\rangle=d\langle L\rangle$ for $L$ being a general non-empty link and O being the trivial link. Also $\langle\mathrm{O}\rangle=1$. Hence $\langle\mathrm{OO} \ldots \mathrm{O}\rangle=d^{m-1}$ where the bracket is over $m$ trivial links that correspond to $n=2 m$ anyons. In particular, when $B_{\vec{a}}$ and $B_{\vec{a}^{\prime}}$ are trivial (corresponding to trivial statistics or involving trivial anyonic braidings) then $\operatorname{tr}\left(B_{\vec{a}}^{t}|\alpha\rangle\langle\alpha| B_{\vec{a}^{\prime}}^{t}\right)=1$, i.e. we obtain the usual quantum walk, with no anyonic effect appearing. 


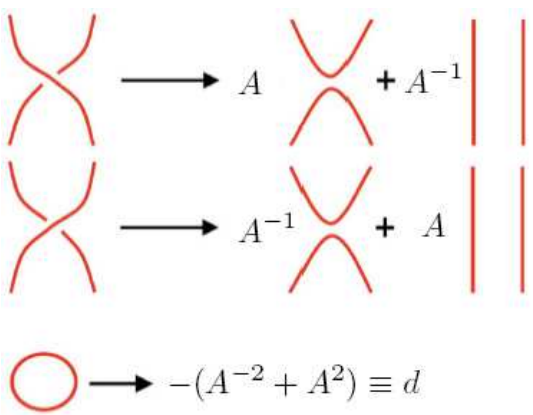

FIG. 5: Method to recursively calculate the Kauffman bracket $\langle L\rangle(A)$ of an unoriented link $L$. All crossing in a projection of the link can be smoothed using the Skein relations and components which are unknots contribute a multiplicative factor of $d$. Normalization is chosen so that $\langle O\rangle=1$.

\section{A. The $\mathbf{S U}(2)$ level $k$ anyonic model}

While the main structures presented here are applicable to general non-Abelian models we present, for concreteness, analytic calculations for the $\mathrm{SU}(2)_{k}$ topological models. These have $A=i e^{i \pi / 2(k+2)}$ so their quantum dimension is $d=2 \cos \left(\frac{\pi}{k+2}\right)$ where $k$ is an integer greater or equal to 2 . All the braiding and fusion matrices of these models are well known [36]. In the following we explicitly calculate the probability distribution in the position of the walker for $t=1,2,3,4$ and 10 number of walker steps.

Let us consider the $t=1$ case. For that walk we use two pairs of anyons canonically ordered on a line and the walker is the third one from the left. Then we have

$$
\left.\sum_{\vec{a}, \vec{a}^{\prime}} \rho_{\vec{a}, \vec{a}^{\prime}}(t=1)\right|_{\text {diag }}=|2\rangle\left\langle 2\left|\operatorname{tr} \mathcal{U} u_{00}^{1} \operatorname{tr} \mathcal{Y}_{00}^{1}+\right| 4\right\rangle\langle 4| \operatorname{tr} \mathcal{U} \mathfrak{l}_{11}^{1} \operatorname{tr} \mathcal{Y}_{11}^{1}
$$

But

$$
\mathcal{U}_{00}^{1}=\mathcal{U}_{11}^{1}=\frac{1}{2}, \text { and } \operatorname{tr} \mathcal{Y}_{00}^{1}=\operatorname{tr} \mathcal{Y}_{11}^{1}=\frac{1}{d}\langle\mathrm{OO}\rangle=1,
$$

so

$$
\left.\sum_{\vec{a}, \vec{a}^{\prime}} \rho_{\vec{a}, \vec{a}^{\prime}}(t=1)\right|_{\text {diag }}=\frac{1}{2} \frac{1}{d}\langle\mathrm{OO}\rangle(|2\rangle\langle 2|+| 4\rangle\langle 4|)=\frac{1}{2}(|2\rangle\langle 2|+| 4\rangle\langle 4|) .
$$

Hence, after one step the walker has equal probability $(1 / 2)$ to be at the two neighboring sites for all types of anyons and no anyonic effects appear.

Let us consider the $t=2$ case. Here we use three pairs of anyons, where the walker is initially located at position $s_{0}=3$. One can evaluate

$$
\operatorname{tr} \mathcal{U}_{\vec{a} \vec{a}^{\prime}}^{2}=\frac{1}{4}
$$

for all relevant pairs $\vec{a} \vec{a}^{\prime}$, i.e. $(0,0)(0,0),(1,1)(1,1),(0,1)(0,1)$ and $(1,0)(1,0)$ while it is zero otherwise. For the trace of braidings we have

$$
\operatorname{tr} \mathcal{Y}_{(0,0)(0,0)}^{2}=\frac{1}{d^{2}}\left\langle\left(b_{1} b_{2}\right)^{\dagger} b_{1} b_{2}\right\rangle=\frac{1}{d^{2}}\langle\mathrm{OOO}\rangle=1
$$

and similarly

$$
\operatorname{tr} \mathcal{Y}_{(1,1)(1,1)}^{2}=\operatorname{tr} \mathcal{Y}_{(0,1)(0,1)}^{2}=\operatorname{tr} \mathcal{Y}_{(1,0)(1,0)}^{2}=1
$$


Hence, we finally have

$$
\left.\sum_{\vec{a}, \vec{a}^{\prime}} \rho_{\vec{a}, \vec{a}^{\prime}}(t=2)\right|_{\text {diag }}=\frac{1}{4}(|1\rangle\langle 1|+| 5\rangle\langle 5|)+\frac{1}{2}|3\rangle\langle 3| .
$$

Again the anyonic character is not apparent at $t=2$ either.

Let as consider the probability distribution for $t=3$ where we use 5 pairs of anyons. The paths $\vec{a} \vec{a}^{\prime}$ that start at the common initial position $s_{0}=5$ and end at the same final position having at the same time the same final coin state are given by $(000)(000),(001)(001),(010)(010),(100)(100),(011)(011),(101)(101),(110)(110),(111),(111)$, $(010)(100),(100)(010),(011)(101)$, and (101)(011). One can see that for all of the paths we have tr $\mathcal{U}_{\vec{a} \vec{a}^{\prime}}^{3}=1 / 8$ apart from the last two $(011)(101)$, and (101)(011) for which $\operatorname{tr} \mathcal{U}_{\vec{a} \vec{a}^{\prime}}^{3}=-1 / 8$. Also for all of the paths we have $\operatorname{tr} \mathcal{Y}_{\vec{a} \vec{a}^{\prime}}^{3}=1$ apart from the last four for which it is

$$
\operatorname{tr}\left(B_{(010)}^{3}{ }^{\dagger}|\alpha\rangle\langle\alpha| B_{(100)}^{3}\right)+\operatorname{tr}\left(B_{(100)}^{3}|\alpha\rangle\langle\alpha| B_{(010)}^{3}\right)=\frac{1}{d}\left(\langle L\rangle+\langle L\rangle^{*}\right)=\frac{2 \cos \left(\frac{2 \pi}{k+2}\right) \cos \left(\frac{3 \pi}{k+2}\right)}{\cos \left(\frac{\pi}{k+2}\right)}
$$

and

$$
\operatorname{tr}\left(B_{(011)}^{3}{ }^{\dagger}|\alpha\rangle\langle\alpha| B_{(101)}^{3}\right)+\operatorname{tr}\left(B_{(101)}^{3}|\alpha\rangle\langle\alpha| B_{(011)}^{3}\right)=\frac{1}{d}\left(\langle L\rangle+\langle L\rangle^{*}\right)=\frac{2 \cos \left(\frac{2 \pi}{k+2}\right) \cos \left(\frac{3 \pi}{k+2}\right)}{\cos \left(\frac{\pi}{k+2}\right)}
$$

where $L$ is the non-trivial link given in Fig. 6 a). Hence, the position distribution is of the form

$$
\left.\sum_{\vec{a}, \vec{a}^{\prime}} \rho_{\vec{a}, \vec{a}^{\prime}}(t=3)\right|_{\text {diag }}=\frac{1}{8}|2\rangle\left\langle 2\left|+\left(\frac{3}{8}+\frac{1}{4} \frac{\cos \left(\frac{2 \pi}{k+2}\right) \cos \left(\frac{3 \pi}{k+2}\right)}{\cos \left(\frac{\pi}{k+2}\right)}\right)\right| 4\right\rangle\left\langle 4\left|+\left(\frac{3}{8}-\frac{1}{4} \frac{\cos \left(\frac{2 \pi}{k+2}\right) \cos \left(\frac{3 \pi}{k+2}\right)}{\cos \left(\frac{\pi}{k+2}\right)}\right)\right| 6\right\rangle\left\langle 6\left|+\frac{1}{8}\right| 8\right\rangle\langle 8| .
$$

The trace of this reduced density matrix is 1 as expected. Nevertheless, it acquires nontrivial contributions from the anyonic nature of the walker and its environment.

For the $t=4$ case we have

$$
\begin{gathered}
\left.\sum_{\vec{a}, \vec{a}^{\prime}} \rho_{\vec{a}, \vec{a}^{\prime}}(t=4)\right|_{\text {diag }}=\frac{1}{16}|1\rangle\left\langle 1\left|+\frac{1}{8}\left(-3 \cos \left(\frac{2 \pi}{k+2}\right)+3 \cos \left(\frac{4 \pi}{k+2}\right)+5\right)\right| 3\right\rangle\langle 3|+ \\
\frac{1}{32}\left(3 \cos \left(\frac{2 \pi}{k+2}\right)-\cos \left(\frac{4 \pi}{k+2}\right)-3 \cos \left(\frac{6 \pi}{k+2}\right)+5\right) \sec ^{2}\left(\frac{\pi}{k+2}\right)|5\rangle\langle 5|+ \\
\frac{1}{16}\left(2 \cos \left(\frac{2 \pi}{k+2}\right)-\cos \left(\frac{4 \pi}{k+2}\right)+1\right) \sec ^{2}\left(\frac{\pi}{k+2}\right)|7\rangle\left\langle 7\left|+\frac{1}{16}\right| 9\right\rangle\langle 9|,
\end{gathered}
$$

where the contributing links are given in Fig. 6(a-d).

(a)

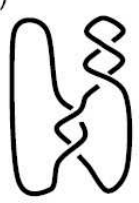

(b)

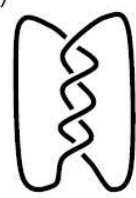

(c)

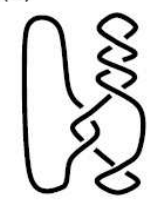

(d)

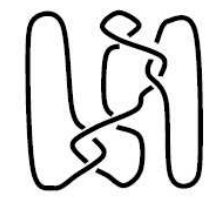

FIG. 6: The links that contribute to the $t=3$ (a) and $t=4$ (a-d) anyonic quantum walks.

We have obtained analytic results for the distribution of the walker for a $t=10$ step walk. This distribution is depicted in Fig. (7) a) as a function of the position of the walker and the level $k$ of the $\mathrm{SU}(2)_{k}$ theory. We notice that 
for large $k$ the distribution, $P(s, t)$, approaches an asymptotic value. This is true for any $t$ we checked. In order to determine the behavior of the walker distribution we compare it to the classical and quantum walks. For that we introduce the distance between the anyonic distribution $P(s, 10)$ and the corresponding standard quantum or classical ones $P_{q, c}(s, 10)$ as $d_{q, c}=\sqrt{\sum_{s}\left|P(s, 10)-P_{q, c}(s, 10)\right|^{2}}$. From Fig. 7 7 b) we see that $d_{q}$ goes to zero for large $k$. For example, for $k>70$ it is $d_{q}<0.01$. Hence, for large $k$ the anyonic walk distributions are very close to ones obtained from the corresponding quantum walks. This is in agreement with the intuition that for $k \rightarrow \infty$ the anyons behave as spin- $1 / 2$ fermions ( $\phi=\pi$ of the Abelian case), which reproduces the standard quantum walk distribution. On the other hand the distance to the classical random walk, $d_{c}$, is closer to zero for small $k$, while in this region $d_{q}$ becomes maximal. From Fig. 7 $(\mathrm{b})$ we see that the minimum of $d_{c}=0.126$ is obtained for $k=6$. Clearly, walks with much larger number of steps is needed in order to conclusively determine the $t$ asymptotic behavior of this anyonic model.

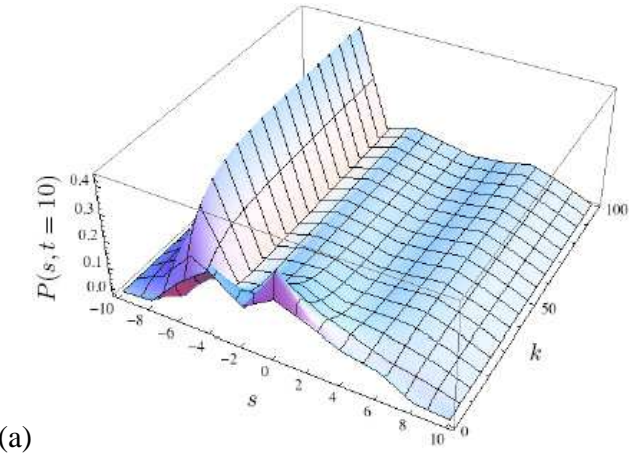

(b)

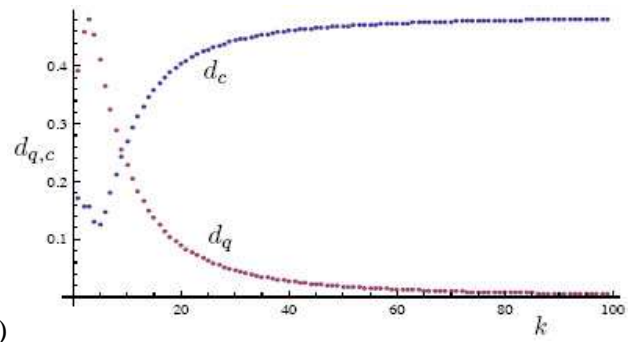

FIG. 7: (a) Distribution of the quantum walker, $P(s, t=10)$, for a $t=10$ step walk as a function of position $s$ and the type of anyonic model indexed by $k$. The origin has been shifted so that the starting point is $s_{0}=0$. (b) The distance $d_{q}$ and $d_{c}$ between the distribution $P(s, t=10)$ and the corresponding quantum $P_{q}(s, 10)$ and classical $P_{c}(s, 10)$, respectively. Clearly, for small $k$ the dispersion is closer to the classical one and for large $k$ it is closer to the quantum one.

It is instructive to consider the decomposition of the anyonic Hilbert space in terms of qubits. As a specific example consider the case of $\mathrm{SU}(2)_{2}$ anyons with labels $1, \sigma, \psi$ representing particles with spin $0, \frac{1}{2}, 1$ respectively. The nontrivial fusion rules are

$$
\sigma \times \sigma=1+\psi, \quad \sigma \times \psi=\sigma, \quad \psi \times \psi=1
$$

and the relevant non-trivial $F$ and $R$ matrices are

$$
F_{\sigma \sigma \sigma}^{\sigma}=\frac{1}{\sqrt{2}}\left(\begin{array}{cc}
1 & 1 \\
1 & -1
\end{array}\right), \quad R_{\sigma \sigma}=\left(\begin{array}{ll}
1 & 0 \\
0 & i
\end{array}\right),
$$

expressed in the basis $\{1, \psi\}$. For the total charge zero superselection sector, the fusion rules require that the only physical fusion basis states are those with $a_{2 k}=\sigma$ for $k=\left[1, \frac{n}{2}-1\right] \cap \mathbb{Z}$. This then gives a fusion space isomorphic to $m=\frac{n}{2}-1$ qubits. The generators of the braid group $\mathcal{B}_{n}$ assume a particularly simple representation in $\mathcal{H}_{\text {fusion }}(n) \simeq$ $\left(\mathbb{C}^{2}\right)^{\otimes m}$

$$
\begin{aligned}
B_{1} & =R \otimes_{j=2}^{m} \mathbf{1}_{2}, \quad B_{2}=B \otimes_{j=2}^{m} \mathbf{1}_{2}, \quad B_{3}=A \otimes_{j=3}^{m} \mathbf{1}_{2} \\
B_{2 k} & =\otimes_{j=1}^{k-1} \mathbf{1}_{2} \otimes B \otimes_{j=k+1}^{m} \mathbf{1}_{2}, \quad B_{2 k+1}=\otimes_{j=1}^{k-1} \mathbf{1}_{2} \otimes A \otimes_{j=k+2}^{m} \mathbf{1}_{2} ; \quad 1<k<m \\
B_{n-3} & =\otimes_{j=1}^{m-2} \mathbf{1}_{2} \otimes A, \quad B_{n-2}=\otimes_{j=1}^{m-1} \mathbf{1}_{2} \otimes B, \quad B_{n-1}=\otimes_{j=1}^{m-1} \mathbf{1}_{2} \otimes R,
\end{aligned}
$$

where

$$
\begin{aligned}
R & =\left(\begin{array}{cc}
1 & 0 \\
0 & i
\end{array}\right), \quad B=\left[F_{\sigma \sigma \sigma}^{\sigma}\right]^{-1} R F_{\sigma \sigma \sigma}^{\sigma}=\frac{1}{\sqrt{2}}\left(\begin{array}{cc}
e^{i \frac{\pi}{4}} & e^{-i \frac{\pi}{4}} \\
e^{-i \frac{\pi}{4}} & e^{i \frac{\pi}{4}}
\end{array}\right), \\
A_{a_{i}}^{a_{i+2}} & =\sum_{x}\left[\left(F_{a_{i} \sigma \sigma}^{a_{i+2}}\right)^{-1}\right]_{x}^{\sigma} R^{x}\left[F_{a_{i} \sigma \sigma}^{a_{i+2}}\right]_{\sigma}^{x} \Rightarrow A=\left(\begin{array}{cccc}
1 & 0 & 0 & 0 \\
0 & i & 0 & 0 \\
0 & 0 & i & 0 \\
0 & 0 & 0 & 1
\end{array}\right) .
\end{aligned}
$$




\section{B. The $D(G)$ quantum double model}

So far we have described quantum walks with non-Abelian anyons corresponding to irreducible representations (irreps) of the quantum group $S U(2)_{k}$. Yet there are other physical theories with anyonic excitations that are described by finite dimensional irreps of quasi-triangular Hopf algebras. As with quantum groups it is possible to find representations of the braid group induced from these algebras which then allow us to compute the entanglement of the fusion degrees of freedom with the walker's spatial degree of freedom. First we write down a general link polynomial $L(z, \bar{z})$ which is a Laurent polynomial in a complex parameter $z$ and its conjugate $\bar{z}$ for oriented links. We demand that $L(z, \bar{z})$ is an invariant under ambient isotopy. Writing the $n$ component link $L=(B)^{\text {Markov }}$ as the Markov trace over a braid word $B=\prod_{k} b_{p_{k}}^{m_{k}} \in \mathcal{B}_{n}$, with $p_{k} \in\{1,2, \ldots, n-1\}, m_{i} \in \mathbb{Z}$, we have [46, 47]

$$
L(z, \bar{z})=(z \bar{z})^{-n / 2}\left(\frac{\bar{z}}{z}\right)^{e(B) / 2} \varphi(B)
$$

where $e(B)=\sum_{k} m_{k}$ (this is the same as the writhe when we orient all components in the same way). Here the quantity $\varphi(B)$ is the Markov trace which satisfies the following properties

$$
\begin{array}{ll}
\text { (II) } \quad \begin{array}{l}
\varphi\left(B b_{n}\right)= \\
\varphi\left(B b_{n}^{-1}\right)=\bar{z} \varphi(B)
\end{array} \quad \forall B \in \mathcal{B}_{n-1} \subset \mathcal{B}_{n}, \\
& \forall B \in \mathcal{B}_{n-1} \subset \mathcal{B}_{n},
\end{array}
$$

where $z=\varphi\left(b_{n}\right), \bar{z}=\varphi\left(b_{n}^{-1}\right)$. As a consequence of these properties, the link invariant satisfies

$$
\begin{array}{ll}
L\left(B B^{\prime}\right)^{\text {Markov }}=L\left(B^{\prime}\right)^{\text {Markov }} \quad \forall B, B^{\prime} \in \mathcal{B}_{n}, \\
L\left(B b_{n}^{ \pm 1}\right)^{\text {Markov }}=L(B)^{\text {Markov }} \quad \forall B \in \mathcal{B}_{n-1} \subset \mathcal{B}_{n} .
\end{array}
$$

Using the additivity of the writhe number it can been shown that for the link invariant $L_{n}$ constructed from $\varphi_{n}$ we have that, $L_{n}\left(\hat{\theta}_{1} \# \hat{\theta}_{2}\right)=L_{i}\left(\hat{\theta}_{1}\right) L_{n-i}\left(\hat{\theta}_{2}\right)$, where $L_{i}($.$) and L_{n-i}($.$) are respectively constructed from \varphi_{i}($.$) and \varphi_{n-i}($.$) .$

There is a procedure to construct the braid group representation of quasi-triangular Hopf algebras but we focus here on a large class given by the quantum double of a finite group $D(G)$. The irreps of $D(G)$ are labelled by the pair $(k, \alpha)$, where $k$ labels a conjugacy class $\mathcal{C}_{k}$ of $G$ and $\alpha$ labels an irrep, with dimension $|\alpha|$, of the centralizer $Z_{k}$ of a representative element $g_{k} \in C_{k}$. The quantum dimension of these irreps (our anyons) is $d[k, \alpha]=\left|\mathcal{C}_{k}\right||\alpha|$. From the representation theory of quantum doubles it is shown [48, 49] that for the invariant above

$$
L(z, \bar{z})=d[k, \alpha]^{n}\left\langle g_{k}\right\rangle_{\alpha}^{-e(B)} \varphi(B), \quad B \in \mathcal{B}_{n}
$$

with

$$
\varphi(B)=\frac{\operatorname{tr}(B)}{(d[k, \alpha])^{n+1}}, \quad z=\frac{\left\langle g_{k}\right\rangle_{\alpha}}{d[k, \alpha]}, \quad z=\frac{\left\langle g_{k}^{-1}\right\rangle_{\alpha}}{d[k, \alpha]}, \quad\left\langle g_{k}^{ \pm 1}\right\rangle_{\alpha}=\frac{\chi_{\alpha}^{k}\left(g_{k}^{ \pm 1}\right)}{|\alpha|}
$$

and $\chi_{\alpha}^{k}$ is the character in the representation $\alpha$.

Consider as an example the symmetric group $G=S_{N}, N \geq 5$, and $C_{2} \subseteq S_{N}$ the conjugacy class of transpositions of $S_{N}$, of order $\left|C_{2}\right|=\frac{1}{2} N(N-1)$ with chosen representative $g_{2}=(N-1 \bar{N})$, and let $Z_{2}=S_{N-2} \times S_{2}$ be the centralizer subgroup of $g_{2}$ where $S_{2}=\left\{I, g_{2}\right\}$. Let $\alpha=1$ labelling the trivial irrep of $Z_{2}$, from which the $(k, \alpha)=(2,1)$ irrep of $D\left(S_{N}\right)$ with dimension $d[2,1]=\frac{1}{2} N(N-1)$ is induced (see [48]). For this irrep $\left\langle g_{k=2}\right\rangle_{\alpha=1}=1$ and (18) becomes $L(B)^{\text {Markov }}=d[2,1]^{n} \varphi(B)$.

Consider the braid group on $n+1$ strands, $\mathcal{B}_{n}$, and a canonical word $B=\left(b_{i_{1}}\right)^{m_{1}}\left(b_{i_{2}}\right)^{m_{2}} \ldots\left(b_{i_{n}}\right)^{m_{n}} \in \mathcal{B}_{n}$. If $\left(i_{1}, i_{2}, \ldots, i_{n}\right)$ is an arbitrary permutation of $(1,2, \ldots, n)$ and $m_{i} \in \mathbb{Z}$, the link polynomial for the trace closure of these words reads

$$
L(B)^{\mathrm{Markov}}=\prod_{i=1}^{n}\left\{1+\frac{2}{3}(N-2)\left[1+2 \cos \left(\frac{2 m_{i} \pi}{3}\right)\right]+\frac{1}{4}(N-2)(N-3)\left[1+(-1)^{m_{i}}\right]\right\} .
$$


By virtue of (16), and by taking for coin initial state $|\psi\rangle$, we have for the diagonal elements of the walker's density matrix the following expression

$$
\begin{aligned}
\left.\sum_{\vec{a}, \vec{a}^{\prime}} \rho_{\vec{a}, \vec{a}^{\prime}}(t)\right|_{\text {diag }}= & \sum_{j=1}^{t} \sum_{\mu_{j}=0}^{j} \sum_{k=1}^{t} \sum_{v_{k}=0}^{k} \delta_{\mu_{t}, v_{t}} \delta_{\mu_{1}, v_{1}} \mathcal{u}_{\vec{\mu}, \vec{v}} \\
& \varphi\left(\left(\prod_{r=1}^{t}\left(\delta_{\mu_{r}, \mu_{r-1}}+\delta_{\mu_{r}, \mu_{r-1}+1}\right) b_{s+\mu_{r}+\mu_{r-1}-r}\right)\left(\prod_{r=1}^{t}\left(\delta_{v_{r-1}, v_{r}}+\delta_{v_{r-1}, v_{r}-1}\right) b_{s+v_{r}+v_{r-1}-r}\right)^{\dagger}\right) \\
& \left|\frac{n+1}{2}+2 \mu_{t}-t\right\rangle\left\langle\frac{n+1}{2}+2 \mu_{t}-t\right|,
\end{aligned}
$$

where $\mathcal{U}_{\mu, v}=\operatorname{tr}\left(\prod_{r=0}^{t}\left(P_{\mu_{r+1}-\mu_{r}} U\right)|\psi\rangle\langle\psi|\left(\prod_{r=0}^{t}\left(P_{v_{r+1}-v_{r}} U\right)\right)^{\dagger}\right)$. Here we take $|\psi\rangle\langle\psi|=| 0\rangle\langle 0|$ and $\rho_{\text {walker }}=$ $\left|\frac{n+1}{2}\right\rangle\left\langle\frac{n+1}{2}\right|$ as the initial coin and walker density matrices, respectively. As we now employed the Markov trace rather than the Plat one the obtained probability distribution corresponds to the modified quantum walker algorithm where we start with $n$ anyonic vacuum pairs, braid all right members of each pair uniformly to the right and then perform the quantum walk just on the $n$ strands corresponding to these right members (see Fig. 4 4 a)). Also the reshuffling matrix is taken to be $U=\frac{1}{\sqrt{2}}\left(\begin{array}{ll}1 & i \\ i & 1\end{array}\right)$. It is worth noting that the same results reported below for the $U$ reshuffling matrix are valid if we had employed the Hadamard matrix $H$, as a straightforward calculation shows.

Next we briefly present the expression for the diagonal part of the walker's density matrix for the first time steps. For step $t=1$, we obtain

$$
\left.\sum_{\vec{a}, \vec{a}^{\prime}} \rho_{\vec{a}, \vec{a}^{\prime}}(t=1)\right|_{\text {diag }}=\frac{1}{2} \varphi\left(B_{2}^{\dagger} B_{2}\right)|2\rangle\left\langle 2\left|+\frac{1}{2} \varphi\left(B_{3}^{\dagger} B_{3}\right)\right| 4\right\rangle\left\langle 4\left|=\frac{1}{2}\right| 2\right\rangle\left\langle 2\left|+\frac{1}{2}\right| 4\right\rangle\langle 4| .
$$

For step $t=2$ we have

$$
\begin{aligned}
\sum_{\vec{a}, \vec{a}^{\prime}} \rho_{\vec{a}, \vec{a}^{\prime}}(t= & 2)\left.\right|_{\text {diag }}=\frac{1}{4}\left\{\varphi\left(\left(B_{1} B_{2}\right)^{\dagger} B_{1} B_{2}\right)|1\rangle\left\langle 1\left|+\varphi\left(\left(B_{4} B_{3}\right)^{\dagger} B_{4} B_{3}\right)\right| 5\right\rangle\langle 5|\right. \\
& \left.+\left[\varphi\left(\left(B_{2}\right)^{\dagger} B_{2}\right)+\varphi\left(\left(B_{3}\right)^{\dagger} B_{3}\right)\right]|3\rangle\langle 3|\right\} \\
= & \frac{1}{4}|1\rangle\left\langle 1\left|+\frac{1}{2}\right| 3\right\rangle\left\langle 3\left|+\frac{1}{4}\right| 5\right\rangle\langle 5| .
\end{aligned}
$$

We notice that the diagonal part of the density matrix given above for the first two steps is common (i.e. independent of $N$ ), to all cases of the symmetric group used i.e. $S_{N}, N \geq 5$. This changes for subsequent steps as we see below.

For step $t=3$ we calculate the expression

$$
\begin{aligned}
\sum_{\vec{a}, \vec{a}^{\prime}} \rho_{\vec{a}, \vec{a}^{\prime}}(t= & 3)\left.\right|_{\text {diag }}=\frac{1}{8}\left\{|2\rangle\left\langle 2\left|+\left(3+\varphi\left(\left(B_{4}^{3}\right)^{\dagger} B_{3}^{2} B_{4}\right)+\varphi\left(\left(B_{3}^{2} B_{4}\right)^{\dagger} B_{4}^{3}\right)\right)\right| 4\right\rangle\langle 4|\right. \\
& \left.+\left(3-\varphi\left(\left(B_{6}^{2} B_{5}\right)^{\dagger} B_{5}^{3}\right)-\varphi\left(\left(B_{5}^{3}\right)^{\dagger} B_{6}^{2} B_{5}\right)\right)|6\rangle\langle 6|+| 8\rangle\langle 8|\right\} \\
= & \frac{1}{8}\left\{|2\rangle\left\langle 2\left|+\left(3+\varphi\left(B_{4}^{-2} B_{3}^{2}\right)+\varphi\left(B_{4}^{2} B_{3}^{-2}\right)\right)\right| 4\right\rangle\langle 4|\right. \\
& \left.+\left(3-\varphi\left(B_{5}^{2} B_{6}^{-2}\right)-\varphi\left(B_{5}^{-2} B_{6}^{2}\right)\right)|6\rangle\langle 6|+| 8\rangle\langle 8|\right\},
\end{aligned}
$$

or in terms of link polynomials 


$$
\begin{aligned}
\sum_{\vec{a}, \vec{a}^{\prime}} \rho_{\vec{a}, \vec{a}^{\prime}}(t= & 3)\left.\right|_{\text {diag }}=\frac{1}{8}\left\{|2\rangle\left\langle 2\left|+\left(3+d[2,1]^{-9} L\left(B_{4}^{-2} B_{3}^{2}\right)+d[2,1]^{-9} L\left(B_{4}^{2} B_{3}^{-2}\right)\right)\right| 4\right\rangle\langle 4|\right. \\
& \left.+\left(3-d[2,1]^{-9} L\left(B_{5}^{2} B_{6}^{-2}\right)-d[2,1]^{-9} L\left(B_{5}^{-2} B_{6}^{2}\right)\right)|6\rangle\langle 6|+| 8\rangle\langle 8|\right\} \\
= & \frac{1}{8}\left\{|2\rangle\left\langle 2\left|+\left(3+2 d[2,1]^{-2}\left[1+\frac{1}{2}(N-2)(N-3)\right]^{2}\right)\right| 4\right\rangle\langle 4|\right. \\
& \left.+\left(3-2 d[2,1]^{-2}\left[1+\frac{1}{2}(N-2)(N-3)\right]^{2}\right)|6\rangle\langle 6|+| 8\rangle\langle 8|\right\},
\end{aligned}
$$

where (18) has been used. By means of (19) we obtain

$$
L\left(B_{5}^{2} B_{6}^{-2}\right)=L\left(B_{5}^{-2} B_{6}^{2}\right)=L\left(B_{4}^{-2} B_{3}^{2}\right)=L\left(B_{4}^{2} B_{3}^{-2}\right)=\left[1+\frac{1}{2}(N-2)(N-3)\right]^{2} d[2,1]^{7}
$$

For the choice $N=5$, the dimension is $d[2,1]=10$, and this yields

$$
\left.\rho(t=3)\right|_{\text {diag }}=\frac{1}{8}\left\{|2\rangle\left\langle 2\left|+\left(3+\frac{8}{25}\right)\right| 4\right\rangle\left\langle 4\left|+\left(3-\frac{8}{25}\right)\right| 6\right\rangle\langle 6|+| 8\rangle\langle 8|\right\} .
$$

Similarly for the $t=4$ step we obtain

$$
\begin{aligned}
\sum_{\vec{a}, \vec{a}^{\prime}} \rho_{\vec{a}, \vec{a}^{\prime}}(t= & 4)\left.\right|_{\text {diag }}=\frac{1}{16}|1\rangle\left\langle 1\left|+\left\{\frac{1}{4}+\frac{3}{8}\left[1+\frac{1}{2}(N-2)(N-3)\right]^{2} d[2,1]^{-2}\right\}\right| 3\right\rangle\langle 3| \\
& +\left\{\frac{3}{8}-\frac{1}{4}\left[1+\frac{1}{2}(N-2)(N-3)\right]^{2} d[2,1]^{-2}\right\}|5\rangle\langle 5| \\
& +\left\{\frac{4}{16}-\frac{2}{16}\left[1+\frac{1}{2}(N-2)(N-3)\right]^{2} d[2,1]^{-2}\right\}|7\rangle\left\langle 7\left|+\frac{1}{16}\right| 9\right\rangle\langle 9|,
\end{aligned}
$$

which for the $N=5$ and $d[2,1]=10$ give the diagonal part

$$
\left.\sum_{\vec{a}, \vec{a}^{\prime}} \rho_{\vec{a}, \vec{a}^{\prime}}(t=4)\right|_{\text {diag }}=\frac{1}{16}|1\rangle\left\langle 1\left|+\frac{31}{100}\right| 3\right\rangle\left\langle 3\left|+\frac{67}{200}\right| 5\right\rangle\left\langle 5\left|+\frac{23}{100}\right| 7\right\rangle\left\langle 7\left|+\frac{1}{16}\right| 9\right\rangle\langle 9| .
$$

Before closing this section some comments are in order. Comparison of the diagonal parts of walkers's density matrix obtained above for the two forms of modelling the braid group representations, i.e. quantum group $S U(2)_{k}$ and symmetric group respectively, shows that the walker occupies the same sites at both cases albeit with different occupation probabilities. This difference in the distribution of occupation probabilities is expected to be a general feature, since by construction these probabilities depend both on the algebraic structure (finite group, quantum group etc.), employed to provide a matrix representation of the braid group, via the associated link polynomials. It is worth mentioning that in the limits $k \rightarrow \infty$ and $N \rightarrow \infty$, both distributions converge to the standard quantum walk as seen in Fig. 8 .

\section{OUTLOOK}

We have described here a simple quantum walk on a line segment with $n$ sites. This is a path graph with vertices given by the locations of $n$ anyons and $n-1$ edges connecting the vertices that correspond to generators of the braid group $B_{n}$. One could imagine anyonic walks on more general graphs by embedding the graph onto a cellular surface of genus $g$. Such a mapping is not unique and the study of such embeddings is the focus of topological graph theory [50]. One could then perform walks with an anyon on this higher genus surface using the representation theory for braid groups on arbitrary manifolds [51]. 


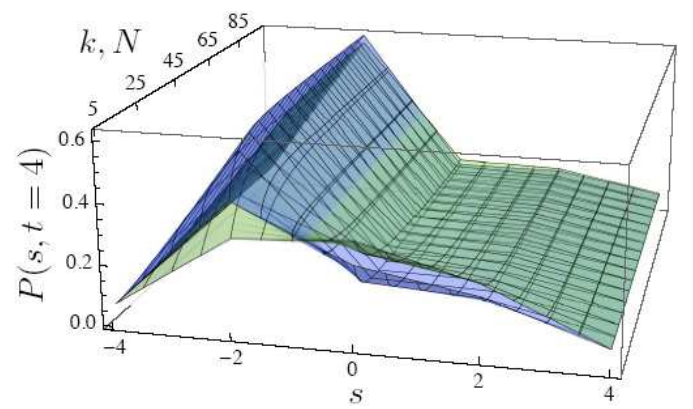

FIG. 8: Combined surface plot for the distribution of a $t=4$ walk in the modified protocol that corresponds to the Markov trace. The blue surface is the distribution for spin-1/2 anyons in the quantum group $S U(2)_{k}$ and the green surface for quantum double anyons in the $(2,1)$ irrep of $D\left(S_{N}\right)$.

There are numerous questions on anyonic quantum walks that have not been addressed yet. The Abelian anyonic quantum walk is easily tractable, both theoretically and numerically, giving rise to a distribution similar to the standard quantum walk. As we have seen one could determine the behavior of non-Abelian quantum walks for a small number of steps. It is a very hard problem to probe any non-Abelian walk for large anyonic chains due to the exponential increase in their Hilbert space dimension. Each additional step of the walker exponentially increases the number of different paths that one needs to consider. On the top of that one needs to evaluate the Kauffman brackets of the corresponding paths in order to determine the final position distribution of the walker. For different types of anyonic models (like $\left.\mathrm{SU}(2)_{2}\right)$ the evaluation of the Jones polynomials is computationally polynomially demanding [52, 53], though still one has to consider the exponential increase in the number of paths. For other models (like $\mathrm{SU}(2)_{k}$ for $k>2$ and $k \neq 4$ ) the Jones polynomial evaluation is believed to be, in general, exponentially hard to compute [53, 54].

Ideally, one is interested in deriving the asymptotic behavior of non-Abelian anyonic quantum walks. The examples we considered here reveal parts of the structure and possibly indicate the asymptotic behavior of certain models. For example, we explicitly considered the $\mathrm{SU}(2)_{k}$ anyonic model for up to $t=10$ number of steps. This gave the following interesting characteristics. For small $k$ the walk appeared to have a distribution close to the classical random walk. For large $k$ the distribution appeared to be similar to a quantum. Even though small $t$ distributions can hardly be conclusive about the large $t$ behavior they provide the exciting possibility of having an anyonic model with intermediate values of $k$ where the variance is in between the classical and the quantum. That would constitute a new paradigm of diffusive behavior. A variety of non-Abelian models needs to be systematically considered theoretically and numerically in order to conclusively understand the asymptotic behavior of non-Abelian walks.

Acknowledgments

This work was supported by the EU grants EMALI and SCALA, UK Engineering and Physical Sciences Research Council and the Royal Society.

[1] N. Shenvi, J. Kempe and K. B. Whaley, Phys. Rev. A 67, 052307 (2003).

[2] A. M. Childs, R. Cleve, E. Deotto, E. Farhi, S. Gutmann and D. A. Spielman, Proc. 35th Annual ACM STOC, pp. 59 (2003).

[3] M. Mohseni, P. Rebentrost, S. Lloyd and A. Aspuru-Guzik, Jour. of Chem. Phys. 129, 174106 (2008).

[4] B. C. Travaglione and G. J. Milburn, Phys. Rev. A 65, 032310 (2002).

[5] W. Dür, R. Raussendorf, V.M. Kendon and H.-J. Briegel, Phys. Rev. A 66, 052319 (2002).

[6] B. C. Sanders, S. D. Bartlett, B. Tregenna and P. L. Knight, Phys. Rev. A 67, 042305 (2003). 
[7] V. M. Kendon and B. C. Sanders, Phys. Rev. A 71, 022307 (2004).

[8] D. Ellinas and I. Smyrnakis, Phys. Rev. A 76, 022333 (2007); Virtual Jour. Quant. Information, 7, 9 (2007)

[9] A. Childs, Phys. Rev. Lett. 102180501 (2009).

[10] R. P. Feynman, Optics News, 11, 11 (1985); A. M. Childs, arXiv:0810.0312, N. Lovett, S. Cooper, M. Everitt, M. Trevers and V. M. Kendon, arXiv:0910.1024

[11] C. A. Ryan, M. Laforest, J. C. Boileau and R. Laflamme, Phys. Rev. A 72, 062317 (2005).

[12] D. Bouwmeester and I. Marzoli, G. P. Karman, W. Schleich and J. P. Woerdman, Phys. Rev. A 61, 013410 (1999).

[13] M. Karski, L. Frster, J.-M. Choi, A. Steffen, W. Alt, D. Meschede and A. Widera, Science 325, p174 (2009).

[14] T. D. Mackay, S. D. Bartlett, L. T. Stephenson and B. C. Sanders, J. Phys. A: Math. Gen. 35, 2745 (2002).

[15] V. M. Kendon and B. Tregenna, Phys. Rev. A 67, 042315 (2003).

[16] A. Bracken, D. Ellinas and I. Smyrnakis, Phys. Rev. A 75, 022322 (2007); Virtual Jour. Quant. Information, 7, 3 (2007)

[17] J. Kempe, Contemp. Phys. 44, 302 (2003).

[18] V. M. Kendon, Phil. Trans. Roy. Soc. A 364, 3407 (2006).

[19] V. M. Kendon, Math. Struct. in Comp. Sci. 17, 1169 (2007).

M. Santha, Theory and Applications of Models of Computation (TAMC08), Xian, April 2008 4978, pp. 31 (2008).

[20] Y. Omar et al., Phys. Rev. A 74, 042304 (2006).

[21] S. Nechaev, Statistics of knots and entangled random walks, Lectures presented at Les Houches 1998 Summer School "Topological Aspects of Low Dimensional Systems", July 7-31, 1998 (NATO Advanced Study Institute, session LXIX: EDP Sciences; Springer, 1999).

[22] E. Farhi and S. Gutmann, Phys. Rev. A 58, 915 (1998).

[23] D. Aharonov, A. Ambainis, J. Kempe and U. Vazirani, Proc. 33rd Annual ACM STOC, pp. 50 ( 2001).

[24] A. Ambainis, E. Bach, A. Nayak, A. Vishwanath and J. Watrous, Proc. 33rd Annual ACM STOC, pp. 60 (2001); A. Nayak and A. Vishwanath, quant-ph/0010117

[25] R. B. Laughlin, Phys. Rev. Lett. 50, 1395 (1983).

[26] F. E. Camino, W. Zhou and V. J. Goldman, Phys. Rev. Lett. 98, 076805 (2007).

[27] T. M. Rice, Science 306, 1142 (2004).

[28] S. Tewari, S. D. Sarma, C. Nayak, C. Zhang, and P. Zoller, Phys. Rev. Lett. 98, 010506 (2007)

[29] L. Fu and C. L. Kane, Phys. Rev. B 76, 045302 (2007).

[30] J. C. Y. Teo, L. Fu, and C. L. Kane, Phys. Rev. B 78, 045426 (2008).

[31] A. Yu. Kitaev, Annals Phys. 303, 2 (2003).

[32] J. K. Pachos, Annals Phys. 322, 1254 (2007).

[33] V. Lahtinen, G. Kells, A. Carollo, T. Stitt, J. Vala and J. K. Pachos, Annals Phys. 323, 2286 (2008).

[34] J. R. Wootton, V. Lahtinen, Z. Wang and J. K. Pachos, Phys. Rev. B 78, 161102(R) (2008).

[35] M. A. Levin and X.-G. Wen, Phys.Rev. B 71, 045110 (2005).

[36] G. K. Brennen and J. K. Pachos, Proc. R. Soc. A 464, 1 (2008).

[37] T. A. Brun, H. A. Carteret, and A. Ambainis, Phys. Rev. Lett. 91, 130602 (2003).

[38] T. A. Brun, H. A. Carteret, and A. Ambainis, Phys. Rev. A 67, 052317 (2003).

[39] T. A. Brun, H. A. Carteret, and A. Ambainis, Phys. Rev. A 67, 032304 (2003).

[40] S. H. Simon, N.E. Bonesteel, M. H. Freedman, N. Petrovic, and L. Hormozi, Phys. Rev. Lett. 96, 070503 (2006).

[41] M. Freedman et al., Com. Math. Phys. 228, 177 (2002).

[42] D. Aharonov, V. Jones and Z. Landau, arXiv:quant-ph/0511096 A pedagogical version of this article is given by S. J. Lomonaco Jr, and L. H. Kauffman, arXiv:quant-ph/0605004.

[43] L. H. Kauffman, Topology 26, 395 (1987).

[44] V. F. R Jones, Bull. Amer. Math. Soc. 12, 103 (1985).

[45] G. Moore and N. Seiberg, Comm. Math. Phys. 123, 177 (1989).

[46] N. Yu. Reshetikhin, L.O.M.I (Leningrand) Preprints E-4-87, E-5-87 (1987); A. N. Kirillov and N. Yu. Reshetikhin, L.O.M.I (Leningrand) Preprints E-9-88 (1988).

[47] R. B. Zang, M. D. Gould and A. J. Bracken, Comm. Math. Phys. 137 (1991) 13.

[48] I. Tsohantjis and M. D. Gould, Bull. Aust. Math. Soc. 49, 177(1994).

[49] M. D. Gould, Bull. Aust. Math. Soc. 48, 275(1993).

[50] J.L. Gross and T.W. Tucker, Topological Graph Theory, Dover Publications, Mineola (2001).

[51] J.S. Birman, Comm. Pure and App. Math. 22, 41 (1969).

[52] W. B. R. Lickorish, Bull. London Math. Soc. 20, 558 (1988).

[53] F. Jaeger, D. L. Vertigan and D. J. A. Welsh, Math. Proc. Camb. Phil. Soc. 108, 35 (1990).

[54] G. Kuperberg, arXiv:0908.0512 